\title{
Lactobacillus fermentum CECT5716 ameliorates high fat diet-induced obesity in mice through modulation of gut microbiota dysbiosis.
}

Jose Alberto Molina-Tijeras ${ }^{1}$, Patricia Diez-Echave ${ }^{1}$, Teresa Vezza ${ }^{1}$, Laura Hidalgo Garcia $^{1}$, Antonio Jesús Ruiz-Malagón ${ }^{1}$, Maria Jesús Rodríguez-Sojoㄹ, Miguel Romero², Iñaki Robles-Vera ${ }^{2}$, Federico Garcia ${ }^{2}$, Julio Plaza-Diaz ${ }^{2}$, Mónica Olivares $^{3}$, Juan Duarte ${ }^{2}$, Maria Elena Rodriguez-Cabezas ${ }^{1}$, Alba Rodríguez-Nogales ${ }^{4}$, and Julio Galvez ${ }^{1}$

${ }^{1}$ CIBER-EHD, Department of Pharmacology, Center for Biomedical Research (CIBM), University of Granada

${ }^{2}$ Instituto de Investigación Biosanitaria de Granada (ibs.GRANADA)

${ }^{3}$ Biosearch Life

${ }^{4} 1$ CIBER-EHD, Department of Pharmacology, Center for Biomedical Research (CIBM), University of Granada

July 23, 2020

\begin{abstract}
Background and Purpose: Obesity is a complex condition where a low-grade chronic inflammation and gut dysbiosis contribute to the development of the related metabolic dysfunctions. Nowadays, its management of such disease implies drastic changes in lifestyle and different anti-obesity drugs. Unfortunately, most of them present limited effectiveness and important side effects. Therefore there is an urgent demand for more effective and safer strategies for obesity management. In this sense, probiotics are emerging as a promising therapy. Different probiotics have demonstrated beneficial effects on this condition, increasing the interest in the development of probiotic treatments. Lactobacillus fermentum CECT5716 has shown anti-inflammatory effects and capacity to modulate microbiota composition in different experimental models. Experimental Approach: L. fermentum CECT5716 (5x10 $\mathrm{CFU} / \mathrm{mice} /$ day $)$ was evaluated in a model of high fat diet-induced obesity in mice. Key results: L. fermentum exerts anti-obesity effects, associated with its anti-inflammatory properties and amelioration endothelial dysfunction and gut dysbiosis. The probiotic restores Akkermansia sp. abundance and reduced Erysipelotrichi class and Clostridium spp presence as well as increased Bacteroides proportion. Conclusion and Implications: This probiotic represents a very interesting approach. Our findings describe, for the first time, the ability of this probiotic to ameliorate experimental obesity through microbiome modulation, affecting different bacteria that have been reported to play a key role in the pathogenesis of obesity. Therefore, this suggests a potential use of L. fermentum CECT5716 in clinical practice, also taking into account that probiotic treatments have demonstrated to be relatively safe and well tolerated.
\end{abstract}

\section{INTRODUCTION}

Metabolic syndrome is one of the public health problems of our time. It affects $25 \%$ of the worldwide population, and its prevalence is constantly increasing (Saklayen, 2018). Metabolic syndrome is characterized by the concurrence of several metabolic dysfunctions including insulin resistance, obesity, hypertension, impaired glucose tolerance, hyperglycemia and dyslipidemia (Eckel, Alberti, Grundy \& Zimmet, 2010). Among them, obesity is considered the central axis of metabolic syndrome (Rogero \& Calder, 2018). This condition is developed as a consequence of an energy imbalance due to an excessive energy intake and 
low expenditure, leading to an abnormal accumulation of lipids in metabolic tissues, mainly adipose tissue and liver (Ding et al., 2010; Esser, Legrand-Poels, Piette, Scheen \& Paquot, 2014). This results in the development of a low-grade systemic inflammatory state, associated with the secretion of pro-inflammatory mediators, such as interleukin (IL)-6 and tumor necrosis factor (TNF)- $\alpha$. Thus, it promotes the recruitment of macrophages to adipose tissues and contributes to the metabolic dysfunctions and obesity-related diseases in these patients (Jang, Han, Kim, Oh, Jang \& Kim, 2019; Lee, Shin \& Choue, 2010). Nowadays, the management of obesity usually implies drastic changes in lifestyle, including dietary restrictions and exercise. In addition, different anti-obesity drugs are available. The most frequently used are phentermine, orlistat, lorcaserin, bupropion and liraglutide, but most of them present limited effectiveness and significant side effects (Saunders, Umashanker, Igel, Kumar \& Aronne, 2018). Therefore, and considering the prevalence of obesity and its co-morbidities, there is a clear demand for more effective and safer strategies for its management.

During the two last decades, different studies have revealed that gut microbiota seems to play an important role in the development of obesity and obesity-associated disorders (Festi, Schiumerini, Eusebi, Marasco, Taddia \& Colecchia, 2014). Obesity has been linked to an altered intestinal microbiota composition, also known as dysbiosis, together with increased gut permeability that promotes bacterial endotoxins translocation, including lipopolysaccharide (LPS), into the systemic circulation, which clearly contributes to the obesity-associated low-grade systemic inflammation (Cani et al., 2007; Teixeira et al., 2012). Considering this, modulation of gut microbiota can result in amelioration of the pathogenic mechanisms involved in obesity, like modulation of the inflammatory response and enhancement of the intestinal barrier function (Gil-Cardoso, Gines, Pinent, Ardevol, Blay \& Terra, 2016). In this context, special attention should be paid to the use of probiotics for the prevention and treatment of obesity-associated metabolic disorders and related diseases, as it has been recently explored (Tenorio-Jimenez, Martinez-Ramirez, Gil \& Gomez-Llorente, 2020).

Probiotics are defined as live microorganisms that confer a health benefit to the host when administered in adequate amounts (Backhed et al., 2004). Among probiotics,Lactobacillus spp. (L. casei strain Shirota (LAB13) ,L. gasseri , L. rhamnosus, and L. plantarum, among others) and Bifidobacterium spp. (mainly B. infantis , B. longum, and B. breve B3 ) are the most relevant in the treatment of metabolic syndrome, demonstrating considerable anti-obesity effects, both in rodents and humans, by modulating weight gain, improving glycemic and lipid metabolism, as well as decreasing insulin resistance (Tenorio-Jimenez, Martinez-Ramirez, Gil \& Gomez-Llorente, 2020). Different mechanisms may be involved in their beneficial effects, including increased short-chain fatty acid (SCFA) production, regulation of bile acid metabolism and host protection from metabolic endotoxemia, most probably through modulation of gut microbiota composition (Daniali, Nikfar \& Abdollahi, 2020). However, a recent systematic review of randomized clinical trials concluded that, up to date, administration of probiotics to patients with metabolic syndrome just produces a discrete improvement, especially when considering their impact on the metabolic profile and the inflammatory biomarkers associated with this condition (Tenorio-Jimenez, Martinez-Ramirez, Gil \& GomezLlorente, 2020). For this reason, there is a great interest in the search of new probiotic-based treatments for human metabolic syndrome that combine efficacy and safety. Among these, the emerging next generation probiotics have started to be studied against these conditions, including Prevotella copri, Christensenella minuta, Parabacteroides goldsteinii, Akkermansia muciniphila or Bacteroides thetaiotaomicron, among others (Chang et al., 2019). However, technological and regulatory limitations can hinder their development for human therapy (El Hage, Hernandez-Sanabria \& Van de Wiele, 2017). Meanwhile, the characterization of other conventional probiotic strains, not previously tested for metabolic syndrome can be an interesting approach. In this context, L. fermentum CECT5716, a probiotic strain originally isolated from human breast milk (Martin et al., 2003) with proven safety and tolerance in infants and mothers (Bond, Morris \& Nassar, 2017), could be of interest. L. fermentum CECT5716 has been shown to display immunomodulatory properties that can be involved in the beneficial effects obtained in experimental models of colitis (RodriguezNogales et al., 2017; Rodríguez-Nogales et al., 2015) and hypertension (Robles-Vera et al., 2018; Toral et al., 2019). Furthermore, these studies highlight the ability of this probiotic to ameliorate gut microbiota dysbio- 
sis and improve the impaired intestinal barrier function, common features of metabolic syndrome (Fasano, 2017; Festi, Schiumerini, Eusebi, Marasco, Taddia \& Colecchia, 2014). Noteworthy, the anti-obesity effects of $L$. fermentum CECT5716 have been previously reported in experimental obesity (Rivero-Gutierrez et al., 2017), although the probiotic was administered with fructooligosaccharides as a synbiotic, being difficult to clearly establish which effects were derived from probiotic administration. Furthermore, other strains of L. fermentum have shown beneficial effects in different experimental models of obesity. Thus, the administration of L. fermentum NCIMB5221 to Zucker diabetic fatty (ZDF) rats improved insulin resistance and lipid metabolism (Tomaro-Duchesneau et al., 2014). Similarly, L. fermentum CQPC05 inhibited high-fat diet (HFD)-induced obesity in mice, an effect associated with an improvement of lipid metabolism (Zhu, Tan, Mu, Yi, Zhou \& Zhao, 2019); while L. fermentum 296 showed protective effects on cardiovascular dysfunction in HFD-treated rats (Cavalcante et al., 2019). Therefore, the aim was to evaluate the effects of the probiotic L. fermentumCECT5716 in a model of diet-induced obesity in mice, and to establish a link between the anti-obesity effect, and its impact on gut dysbiosis, inflammatory status and endothelial dysfunction.

\subsection{Reagents}

All chemicals were purchased from Sigma-Aldrich (Madrid, Spain), unless otherwise stated.

\subsection{Preparation and administration of L. fermentum CECT5716}

The probiotic Lactobacillus fermentum CECT5716 was provided by Biosearch, S.A. (Granada, Spain) and grown in De Man, Rogosa and Sharpe (MRS) media for a period of $24 \mathrm{~h}$ at $37^{\circ} \mathrm{C}$ under anaerobic conditions using the Anaerogen system (Oxoid, Basingstoke, UK). For probiotic treatment, it was suspended using a sterile PBS solution and was diluted to obtain a concentration of $5 \times 10^{9}$ CFUs in $1 \mathrm{ml}$.

\subsection{Animals, diets and experimental design}

The study was carried out in accordance with the 'Guide for the Care and Use of Laboratory Animals' as promulgated by the National Institute of Health, and the protocols approved by the Ethic Committee of Laboratory Animals of the University of Granada (Spain) (Ref. No. 28/03/2016/030). Male C57BL/6J mice (5 weeks old) obtained from Charles River Laboratories (Barcelona, Spain) were housed in a temperature and humidity-controlled facility $\left(22 \pm 1^{\circ} \mathrm{C}, 55 \pm 10 \%\right.$ relative humidity) with a 12-hour light/dark cycle and provided with a free access to tap water. Mice were fed with either a standard chow diet (13\% calories from fat, $20 \%$ calories from protein and 67\% calories from carbohydrate) (Global diet 2014; Harlan Laboratories, Barcelona, Spain) or a HFD in which $60 \%$ of its caloric content was derived from fat (Purified diet 230 HF; Scientific Animal Food \& Engineering, Augy, France). Mice were randomly assigned to different groups $(n=10)$ : control (control diet), obese (HFD) and obese treated (L. fermentum ). Control group received the standard chow while obese groups were fed the HFD. HFD-L. fermentum mice were administered the probiotic L. fermentum at $5 \times 10^{8} \mathrm{CFUs}$ in $100 \mu \mathrm{l} /$ mouse/day and control mice received PBS. The treatment was followed for 11 weeks, controlling regularly animal body weight, food and water intake.

\subsubsection{Glucose tolerance test}

One week before the sacrifice, a glucose tolerance test was performed on mice fasted for $8 \mathrm{~h}$. They received a $50 \%$ glucose solution in water at a dose of $2 \mathrm{~g} / \mathrm{kg}$ of body weight by intraperitoneal injection, and blood was collected from the tail vein at 0, 15, 30,60 and 120 min after injection. Blood glucose was measured using a handheld glucometer (Contour XT, Ascensia Diabetes Care, S.L., Barcelona, Spain).

\subsubsection{Plasma determinations}

At the end of the treatment, mice were sacrificed under isoflurane anesthesia. Blood samples were collected in ice-cold tubes containing heparin and centrifuged at $5000 \mathrm{~g}$ at $4^{\circ} \mathrm{C}$ for $20 \mathrm{~min}$, and the plasma was frozen at -80degC. Plasma glucose, low-density lipoprotein (LDL)-cholesterol, high-density lipoprotein (HDL)-cholesterol and triglycerides concentrations were measured by colorimetric methods using Spinreact kits (Spinreact, S.A., Girona, Spain). Plasma insulin concentrations were quantified using a mouse insulin ELISA kit (Alpco Diagnosis, Salem, NH, USA). Homeostatic model assessment of insulin resistance (HOMA- 
IR) was calculated using the formula: fasting glucose $(\mathrm{mM})$ xfasting insulin $(\mu \mathrm{U} / \mathrm{mL}) / 22.5$. Plasma levels of LPS were measured using a Pierce ${ }^{\mathrm{TM}}$ Chromogenic Endotoxin Quant Kit (Thermo Scientific, Inc., Waltham, MA, USA) following manufacturer's instructions. The plasma samples were pre-treated with a $10 \mathrm{mM} \mathrm{MgCl}_{2}$ solution and Pyrosperse ${ }^{\mathrm{TM}}$ Dispersing Agent (Lonza, Walkersville, MD, USA) in order to remove residual heparin used during blood extraction and fat, respectively. Sterile and pyrogen-free material was always used to guarantee sample and test integrity.

\subsubsection{Morphological variables}

Abdominal and epididymal fat were removed, cleaned, and weighed. Weight indices were calculated by dividing their weights by the tibia length. All tissue samples were frozen in liquid nitrogen and then stored at $-80^{\circ} \mathrm{C}$.

\subsubsection{Histological studies}

Samples of epididymal adipose tissue were fixed in $4 \%$ PFA, embedded in paraffin and $5 \mu \mathrm{m}$-thick sections were taken and stained with hematoxylin and eosin. Adipocyte size was measured and analyzed using Fiji imaging software with the Adiposoft v1.16 plugin.

\subsubsection{Vascular reactivity studies}

Descending thoracic aortic rings were removed from mice and suspended in a wire myograph (model 610M, Danish Myo Technology, Aarhus, Denmark) for isometric tension measurement as previously described by Toral et al. (Toral et al., 2014). Krebs solution (composition in mM: $\mathrm{NaCl} \mathrm{118,} \mathrm{KCl} 4.75, \mathrm{NaHCO}_{3}$ $25, \mathrm{MgSO}_{4} 1.2, \mathrm{CaCl}_{2} 2, \mathrm{KH}_{2} \mathrm{PO}_{4} 1.2$ and glucose 11 ) at $37^{\circ} \mathrm{C}$ was used to fill the organ chamber and was subsequently gassed with $95 \% \mathrm{O}_{2}$ and $5 \% \mathrm{CO}_{2}$ (pH 7.4). Length-tension characteristics were acquired via the myograph software (Myodaq 2.01, Danish Myotechnologies, Denmark) and the aortae were set to a tension of $5 \mathrm{mN}$ and equilibrated during $90 \mathrm{~min}$. Thus, cumulative concentration-response curves to acetylcholine $\left(10^{-9} \mathrm{M}-10^{-5} \mathrm{M}\right)$ were recorded for intact rings pre-contracted by U46619 $\left(10^{-8} \mathrm{M}\right)$ in the absence or in the presence of the selective nicotinamide adenine dinucleotide phosphate (NADPH) oxidase inhibitor VAS2870 $\left(10^{-5} \mathrm{M}\right)$, which was added $30 \mathrm{~min}$ before. Relaxant responses to acetylcholine were expressed as a percentage of pre-contraction.

\subsubsection{NADPH oxidase activity}

NADPH oxidase activity in intact aortic rings was performed by the lucigenin-enhanced chemiluminescence assay, as previously described (Zarzuelo et al., 2011). Briefly, aortic rings from all experimental groups were incubated for 30 minutes at $37^{\circ} \mathrm{C}$ in HEPES-containing physiological salt solution (pH 7.4) containing (in $\mathrm{mM}$ ): $\mathrm{NaCl} 119, \mathrm{HEPES} 20, \mathrm{KCl} 4.6, \mathrm{MgSO}_{4}$ 1, $\mathrm{Na}_{2} \mathrm{HPO}_{4} 0.15, \mathrm{KH}_{2} \mathrm{PO}_{4} 0.4, \mathrm{NaHCO}_{3}$ 1, $\mathrm{CaCl} 21.2$ and glucose 5.5. Aortic production of $\mathrm{O}^{2-}$ was stimulated by addition of NADPH $(100 \mu \mathrm{M})$. Rings were then placed in tubes containing physiological salt solution, with or without NADPH, and lucigenin was injected automatically at a final concentration of $5 \mu \mathrm{mol} / \mathrm{L}$ to withdraw known artefacts when used at higher concentrations. NADPH oxidase activity was determined by measuring luminescence over $200 \mathrm{~s}$ in a scintillation counter (Lumat LB 9507, Berthold, Germany) in 5-s intervals and was measured by subtracting the basal values from those in the presence of NADPH. Vessels were then dried, and their dry weight was determined. NADPH oxidase activity is shown as relative luminescence units (RLU)/min/mg dry aortic ring.

\subsubsection{Analysis of gene expression by RT-qPCR}

Total RNA from aortic rings, liver, fat and colon samples was extracted using RNeasy Mini Kit (Qiagen, Germantown, MD, USA), following the manufacturer's recommendations, and was reverse transcribed using oligo(dT) primers (Promega, Southampton, UK). Real time quantitative PCR amplification and detection was performed on optical-grade 48 well plates in EcoTM Real time PCR System (Illumina, San Diego, CA, USA) with $20 \mathrm{ng}$ of cDNA, the KAPA SYBR@ FAST qPCR Master Mix (Kapa Biosystems, Wilmington, MA, USA) and specific Sigma predesigned primers at their annealing temperature (Table 1). The expression 
levels of the target genes were normalized to that glyceraldehydes 3-phosphate dehydrogenase (Gapdh ) and measured by the comparative $\mathrm{Ct}([?][?] \mathrm{Ct})$.

\subsubsection{DNA extraction and Illumina MiSeq sequencing}

DNA from fecal contents was isolated following the procedure described by Rodriguez-Nogales et al . (Rodriguez-Nogales et al., 2017). Total DNA from stool samples was PCR amplified using primers targeting regions flanking the variable regions 4 through 5 of the bacterial 16S rRNA gene (V4-5), gel purified, and analyzed using multiplexing on the Illumina MiSeq machine. The amplification of a 600-bp sequence in the variable region V4-V5 of the $16 \mathrm{~S}$ rRNA gene was performed using barcoded primers. PCR products were verified visually by running a high-throughput Invitrogen 96-well- E-gel. The PCR reactions from the same samples were pooled in one plate, then cleaned and normalized using the high-throughput Invitrogen SequalPrep 96-well Plate kit. The samples were then pooled to make one library to be quantified fluorometrically before sequencing.

The resulting sequences were completed, quality-filtered, clustered, and taxonomically assigned on the basis of $97 \%$ similarity level against the RDP (Ribosomal Database Project) (Wang, Garrity, Tiedje \& Cole, 2007) by using the QIIME software package (Version 1.9.1) (Knight Lab, San Diego, CA, USA). Sequences were selected to estimate the total bacterial diversity of the DNA samples in a comparable manner and were trimmed to remove barcodes, primers, chimeras, plasmids, mitochondrial DNA and any non-16S bacterial reads and sequences $<150 \mathrm{bp}$.

\subsection{Statistics}

All results are expressed as the mean +- SEM. Differences between means were tested for statistical significance using a one-way analysis of variance (ANOVA) and post-hoc least significance tests. Differences between proportions were analyzed with the chi-squared test. All statistical analyses were carried out with the GraphPad 8 software package (GraphPad Software, Inc., La Jolla, CA, USA), with statistical significance set at $\mathrm{P}<0.05$.

For microbiota evaluation, alpha diversity indices and bacterial abundance data of the different groups were compared using Kruskal-Wallis test followed by pairwise Mann-Whitney U comparison. Resulting p-values were corrected by Bonferroni method. Analysis of $\alpha$-diversity was performed on the output normalized data, which were evaluated using Mothur. LEfSE (linear discriminatory analysis (LDA) effect size) (Version 1.0) was employed to identify biomarkers for both species taxonomic analysis and functional pathways via calculation of the linear discriminant analysis (LDA) score among different phenotype groups. Principal coordinate analysis ( $\mathrm{PCoA}$ ) was performed to identify principal coordinates and visualize $\beta$-diversity in complex multidimensional data of bacteriomes from different groups of mice. Differences in beta-diversity were tested by permutational multivariate analysis of variance (PERMANOVA) using the web-based algorithm tool Microbiome Analyst (Dhariwal, Chong, Habib, King, Agellon \& Xia, 2017; Rodríguez-Nogales et al., 2015). The data are expressed as the mean \pm standard error of the mean (SEM). Experimental data were analyzed in GraphPad Prism 8 (GraphPad Software, Inc., La Jolla, CA, USA) by one-way or two-way ANOVA or Pearson correlation. Data with $\mathrm{P}<0.05$ were considered statistically significant.

Hierarchical clustering and heat maps depicting the metabolic parameters, patterns of abundance and log values were constructed within the "R" statistical software package (version 3.6.0; https://www.r-project.org/) using the "pheatmap", "heatmap.2" and "ggplots" packages. Spearman's correlations of bacterial taxa with metabolic parameters and KEGG metagenomic functions were calculated in the "R" statistical software package (version 3.6.0; https://www.r-project.org/). Co-occurrence networks between taxa and functions were calculated by using the open-source software Gephi (https://gephi.org/) to find differential associations caused by similar alterations in the proportion of different taxa and their predicted functions between different groups of mice. Modularity-based co-occurrence networks were analyzed at a Spearman's correlation cut off 0.7 and p-value $<0.01$; the selected correlation data were imported into the interactive platform, Gephi (version 0.9.2; https://gephi.org), and the following modularity analyses and keystone node identification were conducted within Gephi. 


\section{RESULTS}

\section{L. fermentum CECT5716 reduced weight gain and fat tissue accumulation and improved glucose tolerance test and plasma biochemical profile}

Body weight was recorded in all experimental groups twice a week during the 11-weeks treatment period. As expected, the intake of HFD resulted in a higher mouse body weight gain in the untreated control group when compared to control group receiving the standard diet (Figure 1A). The daily administration of $L$. fermentum to HFD-fed mice significantly reduced weight gain from day 53 onwards, even though food intake was similar in the two groups throughout the experimental period (Figure 1A). Thus, the treatment was able to significantly decrease energy efficiency in comparison with untreated HFD-fed mice, consequently reducing both epididymal and abdominal fat deposits (Figure 1A). The probiotic also had a positive impact on glucose homeostasis as evidenced when glucose tolerance test was performed. Mice fed HFD showed significantly higher glucose level peaks than those fed control diet; however, L. fermentum treatment significantly reduced plasma glucose levels in comparison with HFD control group from 60 min onwards, which resulted in a significant reduction in the area under the curve (AUC) (Figure 1B). Moreover, the histological sections of epididymal fat tissue from untreated HFD-fed mice showed hypertrophy when compared with control diet-fed mice, which was significantly ameliorated after treatment with L. fermentum (Figure 1C).

The plasma biochemical determinations evidenced the obesity-induced alterations in glycemic and lipid profiles. Thus, control HFD-fed mice showed a significant increase of fasted glucose levels compared to nonobese mice, which were significantly reduced by L. fermentum, obtaining similar values to those of the control diet group (Figure 2A). Furthermore, and although no differences were observed among groups when plasma insulin levels were analyzed, an improvement on insulin sensitivity was showed after L. fermentum administration, as evidenced by the reduced HOMA-IR index value compared to control HFD-fed mice (Figure 2A). Similarly, control HFD-fed mice displayed hypercholesterolemia in comparison with control dietfed mice, with higher levels of both low-density lipoprotein (LDL)-cholesterol and high-density lipoprotein (HDL)-cholesterol (data not shown), which resulted in increased LDL/HDL ratio. This ratio was significantly reduced by $L$. fermentum administration to obese mice. In addition, probiotic-treated mice showed reduced plasma triglycerides levels, which were also increased in control HFD-fed mice compared to control diet mice (Figure 2B).

\section{L. fermentum CECT5716 administration reduced the inflammatory status in metabolic tissues}

HFD-diet-induced obesity was associated with a systemic inflammatory status, as evidenced by increased mRNA expression in liver and fat tissues of pro-inflammatory cytokines, including $T \nu \varphi-a$ and $I l-6$, as well as the chemokine monocyte chemotactic protein-1 (Mcp-1), when compared with those mice fed control diet (Figure 3A). L. fermentum administration to obese mice significantly ameliorated all these inflammatory markers, showing in most cases similar mRNA expression to control diet-fed mice (Figure 3A). Likewise, the expression of c-jun N-terminal kinase (Jnk)-1 protein in both tissues was significantly increased in control HFD-fed mice in comparison with the control diet group, whereas probiotic treatment reduced its expression (Figure 3A). Likewise, obese mice also manifested an altered expression of peroxisome proliferator-activated receptor $\alpha(\Pi \pi a \rho a)$ in fat, which was significantly ameliorated after treatment with $L$. fermentum (Figure $3 \mathrm{~B})$.

Furthermore, the impairment in glucose and lipid metabolism in obese mice was associated with a decreased expression of the glucose transporter Glut-4 and AMP-activated protein kinase (Ampk) in liver and fat tissue, which were ameliorated in HFD-fed mice treated with L. fermentum (Figure 3B). Moreover, the expression of Tlr4 in liver and adipose tissues was also altered in control HFD-fed mice, which was significantly restored by the treatment (Figure 3B).

In addition, and in comparison, with control diet-fed mice, HFD intake resulted in an imbalance expression of the adipokines leptin and adiponectin in fat tissue, in combination with a reduced expression of leptin receptor in both liver and fat tissues (Figure 4). The probiotic treatment significantly restored the expression 
of all these genes in fat tissue and increased the expression of leptin receptor in liver (Figure 4).

\section{L. fermentum CECT5716 treatment ameliorated intestinal barrier dysfunction}

The colonic barrier function has been reported to be altered in obesity, as it has been observed in the present study, since the expression of different proteins involved in epithelial integrity was reduced in colon tissue from control HFD-fed mice, including mucins (Muc-1,Muc-2 and Muc-3 ), Zo-1, Occludin and Tff-3 (Figure $5 \mathrm{~A}$ ). The beneficial effects observed with $L$. fermentum in obese mice were associated with the amelioration of the colonic expression of all these proteins, even obtaining similar expressions to those in non-obese control mice (Figure 5A). The improvement in colonic barrier function was associated with lower plasmatic levels of LPS in treated-obese mice when compared with non-treated ones, reaching similar concentrations to control mice (Figure 5B).

\section{L. fermentum CECT5716 supplementation enhanced endothelial function}

In order to evaluate the impact of L. fermentum in obesity-associated cardiovascular dysfunction, endothelium-dependent vasodilator responses to acetylcholine from aortae of mice from different experimental groups were analyzed. HFD-fed mice showed a reduction in the maximal relaxant response in comparison with control mice (Emax values were $59.5 \pm 4.7 \%$ and $71.7 \pm 2.0 \%$ in the HFD and control-diet groups, respectively; $\mathrm{P}<0.05$ ) when the concentration-response curves to acetylcholine were analyzed (Figure $6 \mathrm{~A}$ ). The administration of the probiotic L. fermentum CECT5716 to obese mice significantly improved the altered endothelium-dependent relaxation induced by acetylcholine, obtaining similar $\mathrm{E}_{\max }$ values to those in control diet-fed mice $(72.2 \pm 3.8 \%$ ) (Figure $6 \mathrm{~A}$ ). When the aortic rings were previously incubated with the NADPH oxidase inhibitor VAS2870, no significant differences in the maximal relaxant response were observed among experimental groups. Accordingly, NADPH oxidase activity was significantly increased in the aortic rings from HFD-fed mice compared to control mice, and this activity was reduced in the aortic rings from HFD-fed mice treated with the probiotic (Figure 6B). Moreover, the beneficial impact exerted by the probiotic on vascular function was also associated with reduced expression of pro-inflammatory markers, including $I \lambda-1 \beta$

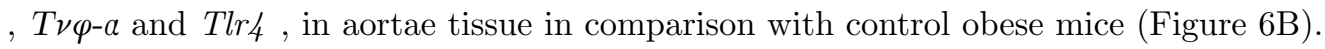

\section{L. fermentum CECT5716 treatment ameliorated the gut dysbiosis}

The effects of $L$. fermentum on the intestinal microbiota were explored since gut dysbiosis plays a key role in metabolic disorders, including obesity. Several ecological features of the gut bacterial communities were evaluated in the three experimental groups by different parameters including Chao1 richness (diversity estimation), Phylogenetic diversity (PD) whole tree (consider the phylogeny to estimate diversity across a tree), Observed OTUs (count of unique OTUs in each sample) and Shannon diversity (a richness and evenness estimator). Microbial richness, evenness and diversity were significantly decreased in the HFD group compared to the control diet group, whereas L. fermentumadministration was able to restore all these ecological parameters to normal values, excepting Shannon index. Although it reached normal values, it did not differ from the control group (Figure 7A). Furthermore, the principal coordinates analysis (PCoA) showed evident differences between control diet- and HFD-fed groups, thus indicating two extremely different gut environments (Figure 7B). Of note, when L. fermentum was administered to obese mice, marked differences could be appreciated in comparison with non-treated obese mice (Figure 7B). To further investigate this remarkable shift in the gut microbial environment after probiotic treatment, the bacteria composition at phylum level was examined (Figure 7C). The $16 \mathrm{~S}$ rDNA analysis revealed that the most abundant phyla were Bacteriodetes (B) andFirmicutes (F) in all experimental groups, although HFD induced a dramatic shift in both phyla when compared to non-obese mice, significantly increasing Firmicutes (from $57.66 \%$ to $78.68 \%$ ) and reducing Bacteroidetes (from $23.72 \%$ to $13.02 \%$ ). This resulted in an increased F/B ratio, which was significantly restored by $L$. fermentum treatment, reaching similar values to control diet-fed mice (Figure 7). Similarly, Verrumicrobia phylum also showed a reduced proportion in HFD-fed mice, which was significantly increased with $L$. fermentum administration (Figure $7 \mathrm{C}$ ). Interestingly, the relative abundance of Erysipelotrichi (class) was enriched in untreated obese mice in comparison with non-obese mice, being this situation ameliorated after probiotic treatment (Figure 7C). 
At genus level, control obese mice revealed a reduced proportion in the sequences in two genera, Bacteroides and Akkermansia, belonging to Bacteriodetes and Verrumicrobia, respectively, and an increase in Clostridium, in comparison with control mice, which was significantly ameliorated after L. fermentum treatment (Figure 7C).

Correlation of major bacteria genera and main obesity-related markers using the non-parametric test of Spearman suggested that beneficial effects observed in L. fermentum -treated mice could be derived from microbiota modulation. In fact, a positive correlation between Akkermansia genus abundance and adipokines and intestinal barrier markers expression, as well as, genus derived fromErysipelotrichaceae and inflammatory markers expression were found (Figure S1). Thus, increased abundance of Akkermansia and reduced presence of Erysipelotrichi (class) observed after L. fermentum treatment could be involved in its beneficial effects.

The PICRUSt (Phylogenetic Investigation of Communities by Reconstruction of Unobserved States) analysis of the KEGG orthologs associated with these bacterial signatures also showed different clusters among the three groups (Figure 8A). LEfSe bar graph indicated that non-obese mice group was characterized by a significantly higher $(\mathrm{p}<0.01)$ proportion of bacterial taxa associated with the energy metabolism, transport and catabolism, metabolism of cofactors and vitamins, replication and repair processes and other glycan degradation (Figure 8A). In contrast, HFD mice harbored a higher $(\mathrm{p}<0.01)$ proportion of bacterial taxa associated with the cellular antigens, biosynthesis of lipopolysaccharides and fatty acids, membrane transporters and metabolism of amino sugar and nucleotide sugar (Figure 8A). When we evaluated the $L$. fermentum treatment in obese mice bacteria related with glycan biosynthesis and metabolism, arginine and proline metabolism, membrane and intracellular structural molecules, nucleotide metabolism or secondary metabolism biosynthesis were overrepresented $(\mathrm{p}<0.01)$, which means that all of these pathways were less represented $(\mathrm{p}<0.01)$ in the other two experimental groups of mice (Figure 8A).

Finally, we estimated the relationship between taxonomic and functional enrichments in each group using the non-parametric test of Spearman's rank correlation. Results, as depicted in the form of hierarchical heat map, showed distinct clusters of positive and negative associations between bacterial taxa and metagenomic functions (Figure 8B). To further simplify these clusters, we extracted the significant correlation subsets (Spearman's rho $>0.7$ and p-value $<0.01$ ) and built separate correlation networks for each experimental group (Figure 8C). Moreover, different co-occurrence networks arrays were observed between non-treated and treated obese mice, confirming previous results.

\section{DISCUSSION}

The treatment with L. fermentum CECT5716 to HFD-fed mice improved HFD-induced obesity, reducing body weight gain, which was associated with an amelioration of glucose and lipid metabolism. The treatment did not significantly modify energy intake, thus discarding any anorexigenic effect, but it showed anti-inflammatory and immunomodulatory properties, as seen before in different experimental models of intestinal inflammation (Rodriguez-Nogales et al., 2017; Rodríguez-Nogales et al., 2015). Indeed, L. fermentum downregulated the expression of pro-inflammatory mediators in HFD-fed mice as well as upregulated the expression of key transcription factors that control adiponectin such as PPAR $\alpha$ (Kim et al., 2015). As in human obesity, this was associated with an improvement in glucose and lipid metabolism, most probably derived from the amelioration of the obesity-associated insulin resistance, as shown by the impact of the probiotic treatment on HOMA-IR values. Moreover, L. fermentumameliorated leptin resistance in liver and adipose tissue as well as restored adiponectin expression in fat contents, which has been previously reported for other probiotics, like different strains of L. plantarum (Choi, Dong, Jeong, Jung, Kim \& Kim, 2019; Kwon et al., 2020).

Obesity-associated insulin resistance is also characterized by impairment of intracellular glucose uptake that is mediated by the insulin-dependent receptor GLUT-4 (Vargas, Podder \& Carrillo Sepulveda, 2020), whose expression is reduced when insulin resistance appears (Shepherd \& Kahn, 1999), similarly to that observed in the present study. L. fermentum also improved insulin resistance, which was associated with an increased expression of Glut-4, ameliorating glycemic levels and glucose utilization by target tissues, as previously 
reported for other probiotics (Chen et al., 2018).

Besides, the obesity-associated inflammatory state is closely related to the development of cardiovascular disease and endothelial dysfunction, in part, mediated by leptin (Huby, Otvos \& Belin de Chantemele, 2016). Conversely, adiponectin has been shown to correlate inversely with arterial hypertension and endothelial dysfunction, since it facilitates the phosphorylation of endothelial nitric oxide synthase (eNOS) enzyme and the subsequent production of nitric oxide (NO), the most effective acetylcholine-induced endothelium-derived relaxing factor in aorta (Adya, Tan \& Randeva, 2015). Additionally, vascular endothelial and smooth muscle cells are also targeting of the pro-inflammatory adipokines TNF- $\alpha$ and IL-6, which, as well, increase expression of pro-inflammatory cytokines, including TNF- $\alpha$ and IL-1 $\beta$, as observed in the present study, and adhesion molecules. This results in the activation of the renin-angiotensin system that leads to inflammation of the vascular walls and development of pre-atheromatous lesions, impairment of vasodilation in humans, most probably through altering the expression and activity of eNOS and NADPH oxidase (Walther et al., 2015). Thus, it modifies the production of $\mathrm{NO}$ and superoxide anion $\left(\mathrm{O}_{2}^{-}\right)$facilitating vascular oxidative stress (Didion, 2017). Interestingly,L. fermentum treatment reduced vascular expression of the pro-inflammatory cytokines TNF- $\alpha$ and IL-1 $\beta$ in obese mice, as well as inhibited the increased NADPH activity in the aortic tissue. This suggests a reduction of ROS production and a higher NO bioavailability, which could promote the restoration of the impaired endothelium-dependent relaxation to acetylcholine, similarly to that reported previously for this probiotic in an experimental model of systemic lupus erythematosus (Toral et al., 2019).

Regarding the involvement of the gut in the pathogenesis of obesity, there is a defect in the intestinal barrier function. This leads to increased gut permeability (Teixeira et al., 2012) that facilitates bacterial components translocation, like LPS, that could reach systemic circulation and provoke metabolic endotoxemia (Cani et al., 2007). LPS may contribute to obesity-associated systemic inflammation upon binding to its main receptor TLR-4, located in immune cells, liver, adipose tissue and skeletal muscle. This promotes the activation of the transcription factor $\mathrm{NF} \varkappa \mathrm{B}$ and subsequent production and release of cytokines, adipokines and ROS, thus altering glucose and lipid homeostasis (Boutagy, McMillan, Frisard \& Hulver, 2016). The present study confirms these observations, since obese mice displayed reduced expression of the colonic markers of epithelial integrity as well as increased LPS plasma levels and up-regulated expression of $T l r-4$ in liver, fat and aorta. Importantly, L. fermentum treatment significantly increased the colonic expression of the different markers involved in gut integrity in obese mice, thus restoring the intestinal barrier function and preventing bacterial components translocation, since it reduced LPS plasma levels and downregulated $T l r-4$ expression. Other probiotics, likeL. sake OK67 (Lim, Jeong, Woo, Han \& Kim, 2016), L. gasseri (Kawano, Miyoshi, Ogawa, Sakai \& Kadooka, 2016)(and Bifidobacterium adolescentis IM38 (Lim \& Kim, 2017) have also been reported to improve intestinal integrity and ameliorate inflammation in obesity in mice. Closely related to the above, the modulation of gut microbiota in obese mice exerted by L. fermentum seems to play a key role. Changes in gut microbial composition, mainly caused by external factors, can result in a dramatic alteration of the symbiotic relationship between gut bacteria and the host and promote the development of metabolic diseases, maybe by facilitating a low-grade inflammation, as mentioned before (Marchesi et al., 2016). In addition, it is well established that gut microbiota composition is altered in obesity (Ley, Backhed, Turnbaugh, Lozupone, Knight \& Gordon, 2005), consisting on an enrichment inFirmicutes (F) as well as a reduction in Bacteroidetes(B), both in humans (De Filippo et al., 2010) and mice (Bagarolli et al., 2017). Increased F/B ratio has been associated with a more efficient hydrolysis of non-digestible polysaccharides in the intestinal lumen, so obese individuals extract more calories and fat from food than lean ones (Backhed et al., 2004). Our study agrees with these observations since HFD-fed mice showed an increased F/B ratio when compared with non-obese mice. However, L. fermentumtreatment was able to modulate gut microbiota composition, restoring the main bacteria phyla to the normal values observed in control diet-fed mice. The PCA analysis showed a clear separation between the clusters, indicating a shift in the gut bacterial composition induced by the probiotic. This amelioration of obesity-associated dysbiosis could be associated with the reduction of energy assimilation and potentially contribute to the beneficial effects observed. Additionally, in obese patients different phyla have been reported to be increased, includingFusobacteria and Proteobacteri a, whereas Verrucobacteria is reduced (Crovesy, Masterson \& Rosado, 
2020). Special attention has been paid to the latter, which includes the bacteria Akkermansia muciniphila , a mucin-degrading bacterium whose abundance is inversely related to body weight and type 2 diabetes in mice (Everard et al., 2013) and humans (Karlsson, Onnerfalt, Xu, Molin, Ahrne \& Thorngren-Jerneck, 2012). Actually, it has been reported that A. muciniphila treatment, or those that promote its abundance, could reverse HFD-induced metabolic disorders (Vezza et al., 2019). In the present study, the proportion of the genus Akkermansia was reduced in untreated obese mice, while increased after L. fermentumtreatment, maybe through an increment in colonic mucins production. This probiotic could become a valid tool to acquire a proper Akkermansia abundance that could have a therapeutic effect in obesity, as demonstrated before (Chang et al., 2019; El Hage, Hernandez-Sanabria \& Van de Wiele, 2017).

Furthermore, it has been reported that Erysipelotrichi, specifically, Clostridium spp, is associated with metabolic syndrome and obesity in humans (Karlsson et al., 2013) and mice (Chakraborti, 2015). Our results confirmed this since Erysipelotrichi class and Clostridium spp abundance were increased in HFD-fed mice while $L$. fermentum treatment reduced significantly their presence in obese mice.

Regarding Bacteroidetes genus, a significant association between HFD and Bacteroides abundance was found, being higher in lean individuals than in obese ones (Castaner et al., 2018). Thus, some strains of Bacteroides , such as B. acidifaciens, have been reported to prevent obesity and improve insulin sensitivity in mice (Yang et al., 2017). Accordingly, in our study, untreated HFD-fed mice manifested a reducedBacteroides proportion in comparison with control diet-fed mice whereas L. fermentum significantly increased it.

Gut metagenomic analysis, in both control diet and HFD mice, has provided some insights into the mechanism of the microbiome to affect weight gain and obesity. Therefore, within Bacteriodetes, lineages rich in genes involved in amino acid metabolism, translation, and nucleotide metabolism, have been found less abundant in HFD mice, while those genes for membrane transport and replication and repair were increased. Similarly, HFD has been also described to modify gene activation on Firmicutes phylum. Membrane transport (mostly ABC transports), transcription and cell motility pathways were increased in abundance, while those with more genes for carbohydrate and energy metabolisms were decreased (Hildebrandt et al., 2009). Since ABC transporters control the transport of a variety of nutrients such as lipids, sugars, peptides and metals, an increase in the number of these transporters could favor energy intake, as seen in HFD-fed mice. Furthermore, a collection of genes involved in intake and assimilation of sugars are also found to be more abundant in microbiome samples from HFD-fed mice, as well as genes for phosphorus metabolism, mainly phosphotransferase systems involved in the uptake and assimilation of sugars (Greenblum, Turnbaugh \& Borenstein, 2012). These results were confirmed in our study, where HFD-fed mice showed enrichment on genes responsible for transport (including ABC transporter), bacterial secretion, motility and sugars assimilation, among others, and were decreased after L. fermentum administration, confirming the potential of this probiotic on the modulation of gut microbiota.

In summary, HFD consumption promotes alterations in gut microbiota that may increase intestinal permeability and LPS translocation, as well as lead to insulin resistance, glucose homeostasis imbalance and systemic low-grade inflammation. However, these obesity-related features were reversed by changes in the gut microbiota profile induced by L. fermentum CECT5716 administration, which suggests a potential use of $L$. fermentum in clinical practice. In conclusion, the probiotic treatment can be an important tool to prevent and treat patients with obesity and metabolic syndrome.

\section{References}

Adya R, Tan BK, \& Randeva HS (2015). Differential effects of leptin and adiponectin in endothelial angiogenesis. Journal of diabetes research 2015: 648239.

Backhed F, Ding H, Wang T, Hooper LV, Koh GY, Nagy A, et al. (2004). The gut microbiota as an environmental factor that regulates fat storage. Proceedings of the National Academy of Sciences of the United States of America 101: 15718-15723.

Bagarolli RA, Tobar N, Oliveira AG, Araujo TG, Carvalho BM, Rocha GZ, et al. (2017). Probiotics 
modulate gut microbiota and improve insulin sensitivity in DIO mice. The Journal of nutritional biochemistry 50: $16-25$.

Bond DM, Morris JM, \& Nassar N (2017). Study protocol: evaluation of the probiotic Lactobacillus Fermentum CECT5716 for the prevention of mastitis in breastfeeding women: a randomised controlled trial. BMC pregnancy and childbirth 17: 148.

Boutagy NE, McMillan RP, Frisard MI, \& Hulver MW (2016). Metabolic endotoxemia with obesity: Is it real and is it relevant? Biochimie 124: 11-20.

Cani PD, Amar J, Iglesias MA, Poggi M, Knauf C, Bastelica D, et al. (2007). Metabolic endotoxemia initiates obesity and insulin resistance. Diabetes 56:1761-1772.

Castaner O, Goday A, Park YM, Lee SH, Magkos F, Shiow STE, et al. (2018). The Gut Microbiome Profile in Obesity: A Systematic Review. International journal of endocrinology 2018: 4095789.

Cavalcante RGS, de Albuquerque TMR, de Luna Freire MO, Ferreira GAH, Carneiro Dos Santos LA, Magnani M, et al. (2019). The probiotic Lactobacillus fermentum 296 attenuates cardiometabolic disorders in high fat diet-treated rats. Nutrition, metabolism, and cardiovascular diseases : NMCD 29: 1408-1417.

Crovesy L, Masterson D, \& Rosado EL (2020). Profile of the gut microbiota of adults with obesity: a systematic review. European journal of clinical nutrition.

Chakraborti CK (2015). New-found link between microbiota and obesity. World journal of gastrointestinal pathophysiology 6: 110-119.

Chang CJ, Lin TL, Tsai YL, Wu TR, Lai WF, Lu CC, et al. (2019). Next generation probiotics in disease amelioration. Journal of food and drug analysis 27: 615-622.

Chen LH, Chen YH, Cheng KC, Chien TY, Chan CH, Tsao SP, et al. (2018). Antiobesity effect of Lactobacillus reuteri 263 associated with energy metabolism remodeling of white adipose tissue in highenergy-diet-fed rats. The Journal of nutritional biochemistry 54: 87-94.

Choi WJ, Dong HJ, Jeong HU, Jung HH, Kim YH, \& Kim TH (2019). Antiobesity Effects of Lactobacillus plantarum LMT1-48 Accompanied by Inhibition of Enterobacter cloacae in the Intestine of Diet-Induced Obese Mice. Journal of medicinal food 22: 560-566.

Daniali M, Nikfar S, \& Abdollahi M (2020). A brief overview on the use of probiotics to treat overweight and obese patients. Expert review of endocrinology \& metabolism 15: 1-4.

De Filippo C, Cavalieri D, Di Paola M, Ramazzotti M, Poullet JB, Massart S, et al. (2010). Impact of diet in shaping gut microbiota revealed by a comparative study in children from Europe and rural Africa. Proceedings of the National Academy of Sciences of the United States of America 107:14691-14696.

Dhariwal A, Chong J, Habib S, King IL, Agellon LB, \& Xia J (2017). MicrobiomeAnalyst: a web-based tool for comprehensive statistical, visual and meta-analysis of microbiome data. Nucleic acids research 45: W180-W188.

Didion SP (2017). Unraveling the Role and Complexities of Inflammation in Hypertension. Hypertension 70: 700-702.

Ding S, Chi MM, Scull BP, Rigby R, Schwerbrock NM, Magness S, et al. (2010). High-fat diet: bacteria interactions promote intestinal inflammation which precedes and correlates with obesity and insulin resistance in mouse. PloS one 5: e12191.

Eckel RH, Alberti KG, Grundy SM, \& Zimmet PZ (2010). The metabolic syndrome. The lancet 375:181-183.

El Hage R, Hernandez-Sanabria E, \& Van de Wiele T (2017). Emerging Trends in "Smart Probiotics": Functional Consideration for the Development of Novel Health and Industrial Applications. Frontiers in microbiology 8: 1889. 
Esser N, Legrand-Poels S, Piette J, Scheen AJ, \& Paquot N (2014). Inflammation as a link between obesity, metabolic syndrome and type 2 diabetes. Diabetes research and clinical practice 105: 141-150.

Everard A, Belzer C, Geurts L, Ouwerkerk JP, Druart C, Bindels LB, et al. (2013). Cross-talk between Akkermansia muciniphila and intestinal epithelium controls diet-induced obesity. Proceedings of the National Academy of Sciences of the United States of America 110: 9066-9071.

Fasano A (2017). Gut permeability, obesity, and metabolic disorders: who is the chicken and who is the egg? The American journal of clinical nutrition 105: 3-4.

Festi D, Schiumerini R, Eusebi LH, Marasco G, Taddia M, \& Colecchia A (2014). Gut microbiota and metabolic syndrome. World journal of gastroenterology 20: 16079-16094.

Gil-Cardoso K, Gines I, Pinent M, Ardevol A, Blay M, \& Terra X (2016). Effects of flavonoids on intestinal inflammation, barrier integrity and changes in gut microbiota during diet-induced obesity. Nutrition research reviews 29:234-248.

Greenblum S, Turnbaugh PJ, \& Borenstein E (2012). Metagenomic systems biology of the human gut microbiome reveals topological shifts associated with obesity and inflammatory bowel disease. Proceedings of the National Academy of Sciences of the United States of America 109: 594-599.

Hildebrandt MA, Hoffmann C, Sherrill-Mix SA, Keilbaugh SA, Hamady M, Chen YY, et al. (2009). High-fat diet determines the composition of the murine gut microbiome independently of obesity. Gastroenterology 137: 1716-1724 e1711-1712.

Huby AC, Otvos L, Jr., \& Belin de Chantemele EJ (2016). Leptin Induces Hypertension and Endothelial Dysfunction via Aldosterone-Dependent Mechanisms in Obese Female Mice. Hypertension 67: 1020-1028.

Jang HM, Han SK, Kim JK, Oh SJ, Jang HB, \& Kim DH (2019). Lactobacillus sakei Alleviates High-FatDiet-Induced Obesity and Anxiety in Mice by Inducing AMPK Activation and SIRT1 Expression and Inhibiting Gut Microbiota-Mediated NF-kappaB Activation. Molecular nutrition \& food research 63:e1800978.

Karlsson CL, Onnerfalt J, Xu J, Molin G, Ahrne S, \& Thorngren-Jerneck K (2012). The microbiota of the gut in preschool children with normal and excessive body weight. Obesity 20: 2257-2261.

Karlsson FH, Tremaroli V, Nookaew I, Bergstrom G, Behre CJ, Fagerberg B, et al. (2013). Gut metagenome in European women with normal, impaired and diabetic glucose control. Nature 498: 99-103.

Kawano M, Miyoshi M, Ogawa A, Sakai F, \& Kadooka Y (2016). Lactobacillus gasseri SBT2055 inhibits adipose tissue inflammation and intestinal permeability in mice fed a high-fat diet. Journal of nutritional science 5: e23.

Kim AY, Park YJ, Pan X, Shin KC, Kwak SH, Bassas AF, et al. (2015). Obesity-induced DNA hypermethylation of the adiponectin gene mediates insulin resistance. Nature communications 6: 7585 .

Kwon J, Kim B, Lee C, Joung H, Kim BK, Choi IS, et al. (2020). Comprehensive amelioration of high-fat diet-induced metabolic dysfunctions through activation of the PGC-1alpha pathway by probiotics treatment in mice. PloS one 15: e0228932.

Lee IS, Shin G, \& Choue R (2010). Shifts in diet from high fat to high carbohydrate improved levels of adipokines and pro-inflammatory cytokines in mice fed a high-fat diet. Endocrine journal 57: 39-50.

Ley RE, Backhed F, Turnbaugh P, Lozupone CA, Knight RD, \& Gordon JI (2005). Obesity alters gut microbial ecology. Proceedings of the National Academy of Sciences of the United States of America 102: 11070-11075.

Lim SM, Jeong JJ, Woo KH, Han MJ, \& Kim DH (2016). Lactobacillus sakei OK67 ameliorates high-fat diet-induced blood glucose intolerance and obesity in mice by inhibiting gut microbiota lipopolysaccharide production and inducing colon tight junction protein expression. Nutrition research 36: 337-348. 
Lim SM, \& Kim DH (2017). Bifidobacterium adolescentis IM38 ameliorates high-fat diet-induced colitis in mice by inhibiting NF-kappaB activation and lipopolysaccharide production by gut microbiota. Nutrition research 41: 86-96.

Marchesi JR, Adams DH, Fava F, Hermes GD, Hirschfield GM, Hold G, et al. (2016). The gut microbiota and host health: a new clinical frontier. Gut 65: 330-339.

Martin R, Langa S, Reviriego C, Jiminez E, Marin ML, Xaus J, et al. (2003). Human milk is a source of lactic acid bacteria for the infant gut. The Journal of pediatrics 143: 754-758.

Rivero-Gutierrez B, Gamez-Belmonte R, Suarez MD, Lavin JL, Aransay AM, Olivares M, et al. (2017). A synbiotic composed of Lactobacillus fermentum CECT5716 and FOS prevents the development of fatty acid liver and glycemic alterations in rats fed a high fructose diet associated with changes in the microbiota. Molecular nutrition \& food research 61 .

Robles-Vera I, Toral M, de la Visitacion N, Sanchez M, Romero M, Olivares M, et al. (2018). The Probiotic Lactobacillus fermentum Prevents Dysbiosis and Vascular Oxidative Stress in Rats with Hypertension Induced by Chronic Nitric Oxide Blockade. Molecular nutrition \& food research 62:e1800298.

Rodriguez-Nogales A, Algieri F, Garrido-Mesa J, Vezza T, Utrilla MP, Chueca N, et al. (2017). Differential intestinal anti-inflammatory effects of Lactobacillus fermentum and Lactobacillus salivarius in DSS mouse colitis: impact on microRNAs expression and microbiota composition. Molecular nutrition \& food research 61.

Rodríguez-Nogales A, Algieri F, Vezza T, Garrido-Mesa N, Olivares M, Comalada M, et al. (2015). The viability of Lactobacillus fermentum CECT5716 is not essential to exert intestinal anti-inflammatory properties. Food \& Function 6:1176-1184.

Rogero MM, \& Calder PC (2018). Obesity, Inflammation, Toll-Like Receptor 4 and Fatty Acids. Nutrients 10.

Saklayen MG (2018). The Global Epidemic of the Metabolic Syndrome. Current hypertension reports 20: 12.

Saunders KH, Umashanker D, Igel LI, Kumar RB, \& Aronne LJ (2018). Obesity Pharmacotherapy. The Medical clinics of North America 102: 135-148.

Shepherd PR, \& Kahn BB (1999). Glucose transporters and insulin action-implications for insulin resistance and diabetes mellitus. The New England journal of medicine 341: 248-257.

Teixeira TF, Souza NC, Chiarello PG, Franceschini SC, Bressan J, Ferreira CL, et al. (2012). Intestinal permeability parameters in obese patients are correlated with metabolic syndrome risk factors. Clinical nutrition 31:735-740.

Tenorio-Jimenez C, Martinez-Ramirez MJ, Gil A, \& Gomez-Llorente C (2020). Effects of Probiotics on Metabolic Syndrome: A Systematic Review of Randomized Clinical Trials. Nutrients 12.

Tomaro-Duchesneau C, Saha S, Malhotra M, Jones ML, Labbe A, Rodes L, et al. (2014). Effect of orally administered L. fermentum NCIMB 5221 on markers of metabolic syndrome: an in vivo analysis using ZDF rats. Applied microbiology and biotechnology 98: 115-126.

Toral M, Gómez-Guzmán M, Jiménez R, Romero M, Sánchez M, Utrilla MP, et al. (2014). The probiotic Lactobacillus coryniformis CECT5711 reduces the vascular pro-oxidant and pro-inflammatory status in obese mice. Clinical Science 127:33-45.

Toral M, Robles-Vera I, Romero M, de la Visitacion N, Sanchez M, O'Valle F, et al. (2019). Lactobacillus fermentum CECT5716: a novel alternative for the prevention of vascular disorders in a mouse model of systemic lupus erythematosus. FASEB journal : official publication of the Federation of American Societies for Experimental Biology 33: 10005-10018. 
Vargas E, Podder V, \& Carrillo Sepulveda MA (2020). Physiology, Glucose Transporter Type 4 (GLUT4). In StatPearls. Treasure Island (FL).

Vezza T, Rodriguez-Nogales A, Algieri F, Garrido-Mesa J, Romero M, Sanchez M, et al. (2019). The metabolic and vascular protective effects of olive (Olea europaea L.) leaf extract in diet-induced obesity in mice are related to the amelioration of gut microbiota dysbiosis and to its immunomodulatory properties. Pharmacological research 150: 104487.

Walther G, Obert P, Dutheil F, Chapier R, Lesourd B, Naughton G, et al. (2015). Metabolic syndrome individuals with and without type 2 diabetes mellitus present generalized vascular dysfunction: cross-sectional study. Arteriosclerosis, thrombosis, and vascular biology 35:1022-1029.

Wang Q, Garrity GM, Tiedje JM, \& Cole JR (2007). Naive Bayesian classifier for rapid assignment of rRNA sequences into the new bacterial taxonomy. Applied and environmental microbiology 73: 5261-5267.

Yang JY, Lee YS, Kim Y, Lee SH, Ryu S, Fukuda S, et al. (2017). Gut commensal Bacteroides acidifaciens prevents obesity and improves insulin sensitivity in mice. Mucosal immunology 10: 104-116.

Zarzuelo MJ, Jiménez R, Galindo P, Sánchez M, Nieto A, Romero M, et al. (2011). Antihypertensive effects of peroxisome proliferator-activated receptor- $\beta$ activation in spontaneously hypertensive rats. Hypertension 58: 733-743.

Zhu K, Tan F, Mu J, Yi R, Zhou X, \& Zhao X (2019). Anti-Obesity Effects of Lactobacillus fermentum CQPC05 Isolated from Sichuan Pickle in High-Fat Diet-Induced Obese Mice through PPAR-alpha Signaling Pathway. Microorganisms 7.

Table 1. Primer sequences used in RT-qPCR assays

\begin{tabular}{|c|c|c|}
\hline Gene & Organism & Annealing $\mathrm{T}^{\circ} \mathrm{C}$ \\
\hline Gapdh & Mouse & $\begin{array}{l}\text { FW: CCATCAC- } \\
\text { CATCTTCCAGGAG } \\
\text { RV: } \\
\text { CCTGCTTCACCACCTTCTTG }\end{array}$ \\
\hline Adiponectin & Mouse & $\begin{array}{l}\text { FW: GATGGCAGA- } \\
\text { GATGGCACTCC RV: } \\
\text { CTTGCCAGTGCTGCCGTCAT }\end{array}$ \\
\hline Ampk & Mouse & $\begin{array}{l}\text { FW: GACTTC- } \\
\text { CTTCACAGCCTCATC } \\
\text { RV: } \\
\text { CGCGCGACTATCAAAGACATACG }\end{array}$ \\
\hline Glut4 & Mouse & $\begin{array}{l}\text { FW: GAGAATACAGC- } \quad 62 \\
\text { TAGGACCAGTG RV: } \\
\text { TCTTATTGCAGCAGCGCCTGAG }\end{array}$ \\
\hline Il- $1 \beta$ & Mouse & $\begin{array}{l}\text { FW: TGATGAGAAT- } \quad 60 \\
\text { GACCTCTTCT RV: } \\
\text { CTTCTTCAAAGATGAAGGAAA }\end{array}$ \\
\hline Il-6 & Mouse & $\begin{array}{l}\text { FW: TAGTCCTTCC- } \quad 60 \\
\text { TACCCCAATTTCC RV: } \\
\text { TTGGTCCTTAGCCACTCCTTCC }\end{array}$ \\
\hline Jnk-1 & Mouse & $\begin{array}{l}\text { FW: GATTTTG- } \\
\text { GACTGGCGAGGACT } \\
\text { RV: } \\
\text { TAGCCCATGCCGAGAATGA }\end{array}$ \\
\hline
\end{tabular}




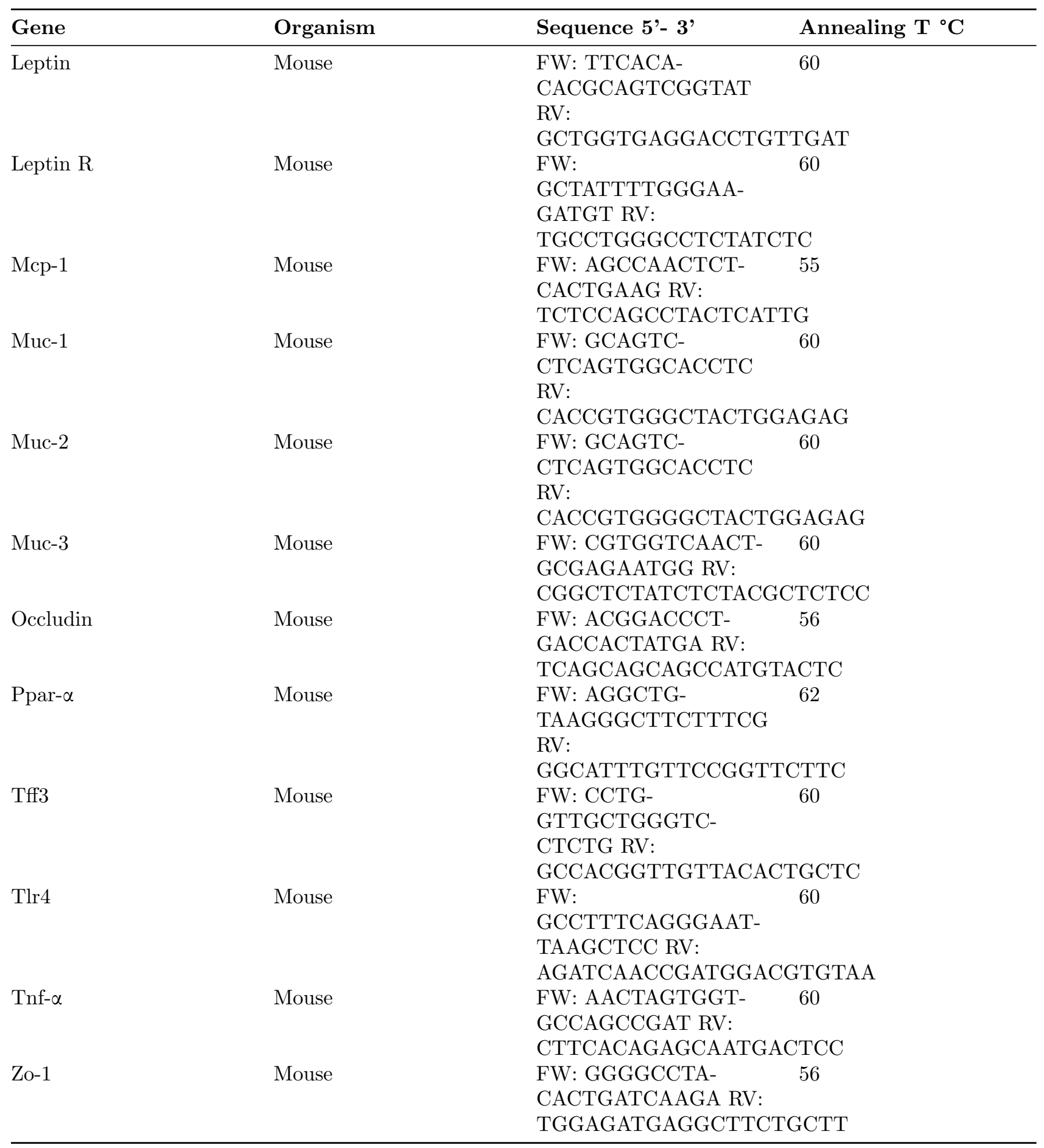

\section{FIGURE LEGENDS}

Figure 1. Effects of L. fermentum CECT5716 administration on a) morphological changes (body weight evolution, energy intake, energy efficiency, abdominal and epididymal fat); b) Glucose tolerance test and area under the curve (AUC) and c) Epididymal adipose tissue, analysed by hematoxylin and eosin staining 
$($ scale bar $=50 \mu \mathrm{m})$ and on adipocyte area. Data are expressed as means \pm SEM $(\mathrm{n}=10)$. Groups with different letter statistically differ $(\mathrm{P}<0.05)$.

Figure 2. a) Basal Glucose, insulin levels and HOMA-IR index, and; b) LDL/HDL ratio and Triglycerides plasma levels in control and high fat diet (HFD)-fed mice. Data are expressed as means \pm SEM $(n=10)$. Groups with different letter statistically differ $(\mathrm{P}<0.05)$.

Figure 3. Effects of $L$. fermentum CECT5716 administration on liver and/or fat gene expression of a) $\mathrm{Il}-6$

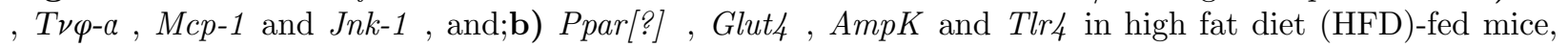
analysed by real time qPCR. Data are expressed as means \pm SEM $(n=10)$. Groups with different letter statistically differ $(\mathrm{P}<0.05)$.

Figure 4. Effects of L. fermentum CECT5716 administration on fat gene expression of Leptin andAdinopectin, and on fat and liver gene expression of Leptin $R$ in high fat diet (HFD)-fed mice, analysed by real time $\mathrm{qPCR}$. Data are expressed as means \pm SEM $(\mathrm{n}=10)$. Groups with different letter statistically differ $(\mathrm{P}<0.05)$.

Figure 5. Effects of L. fermentum CECT5716 administration on a) gene expression of intestinal barrier integrity markers Muc-1, Muc-2 , Muc-3, Zo-1 ,Occludin and Tff-3 in high fat diet (HFD)-fed mice, analysed by real time $\mathrm{qPCR}$; b) plasma endotoxin concentrations $(\mathrm{EU} / \mathrm{mL}$, endotoxin units $/ \mathrm{mL})$. Data are expressed as means \pm SEM $(\mathrm{n}=10)$. Groups with different letter statistically differ $(\mathrm{P}<0.05)$.

Figure 6. Effects of L. fermentum CECT5716 administration on endothelial function: a)endotheliumdependent relaxation in the absence or the presence NADPH oxidase inhibitor VAS2870; b) aortic NADPH activity and gene expression of Tlr4, Tnf- $\alpha$ and Il- $1 \beta$ in control and high fat diet (HFD)-fed mice. Data are expressed as means \pm SEM $(\mathrm{n}=10)$. Groups with different letter statistically differ $(\mathrm{P}<0.05)$.

Figure 7. Impact of L. fermentum CECT5716 administration on a) microbiome diversity (Chao1, PD whole tree, Observed OTUs and Shannon index); b) Beta-diversity by principal coordinate analysis score plot, and c) bacterial community (phyla, class and genus) and the F/B ratio. Data are expressed as means \pm SEM. Groups with different letter statistically differ $(\mathrm{P}<0.05)$.

Figure S1. Heat-map of taxon that were most significantly different in abundance and main obesity-related markers. Correlation using non-parametric test of Spearman.

Figure 8. a) Metagenomic functional features predicted by PICRUSt (Phylogenetic Investigation of Communities by Reconstruction of Unobserved States) that were differentially abundant and drove differences in non-obese mice (control mice), HFD-group and in the treatment obese mice (L. fermentum group); b)Correlation between bacterial taxa and functional features in each group; and c) Correlation networks for control mice, HFD-group and L. fermentum treated mice.

\section{Hosted file}

Figure 1.pdf available at https://authorea.com/users/345589/articles/471772-lactobacillusfermentum-cect5716-ameliorates-high-fat-diet-induced-obesity-in-mice-through-modulationof-gut-microbiota-dysbiosis 
Figure 2

a)
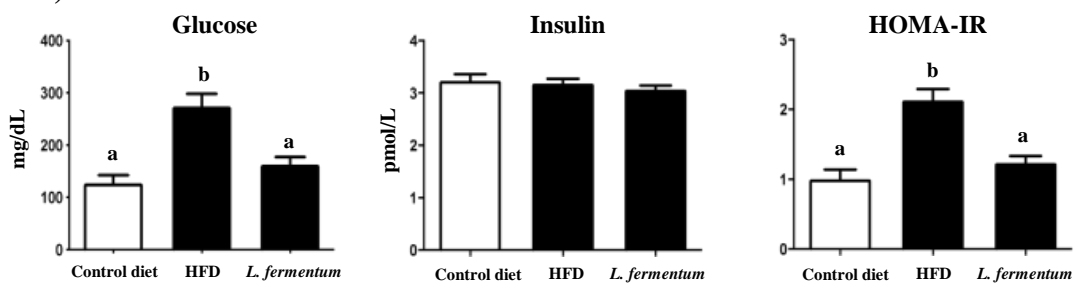

b)
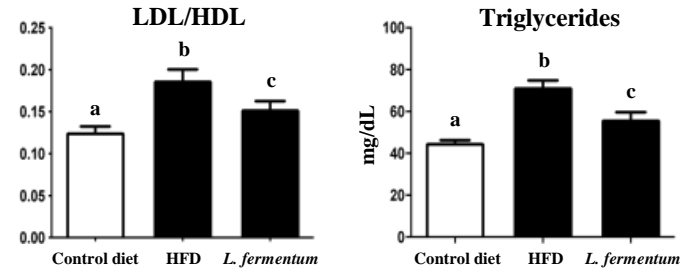
Figure 3

a)
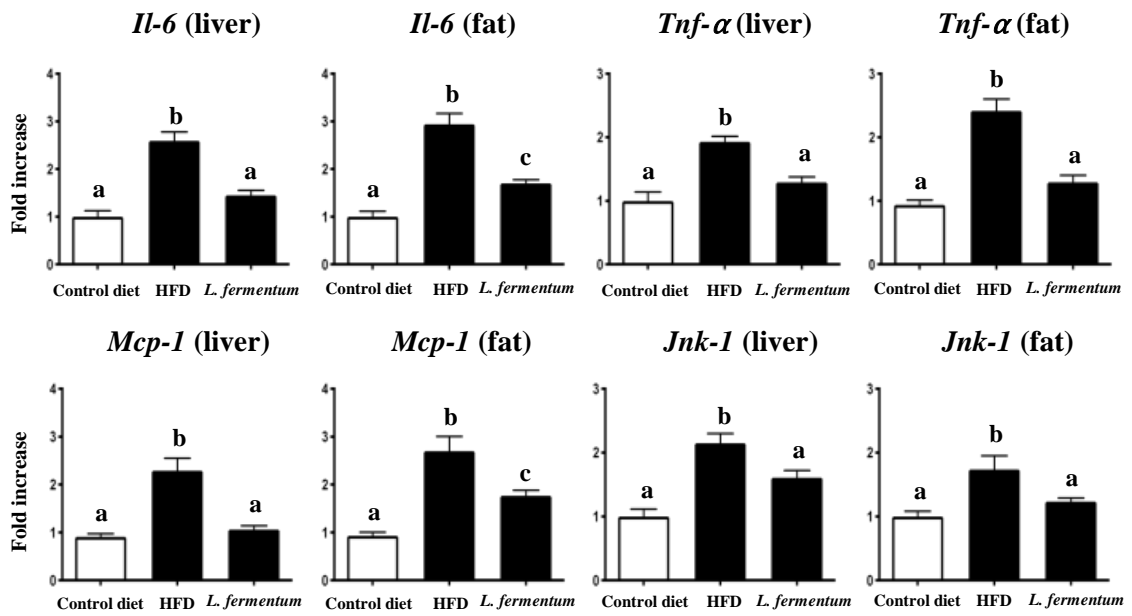

b)

$\operatorname{Ppar} \alpha$ (fat)

Glut4 (fat)

Ampk (liver)

Ampk (fat)
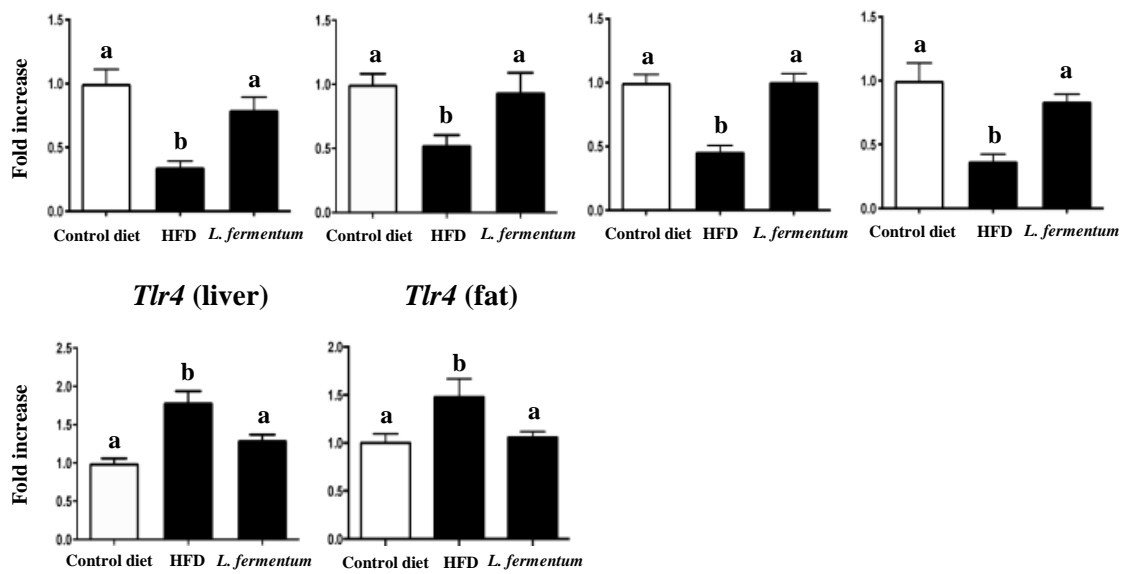
Figure 4
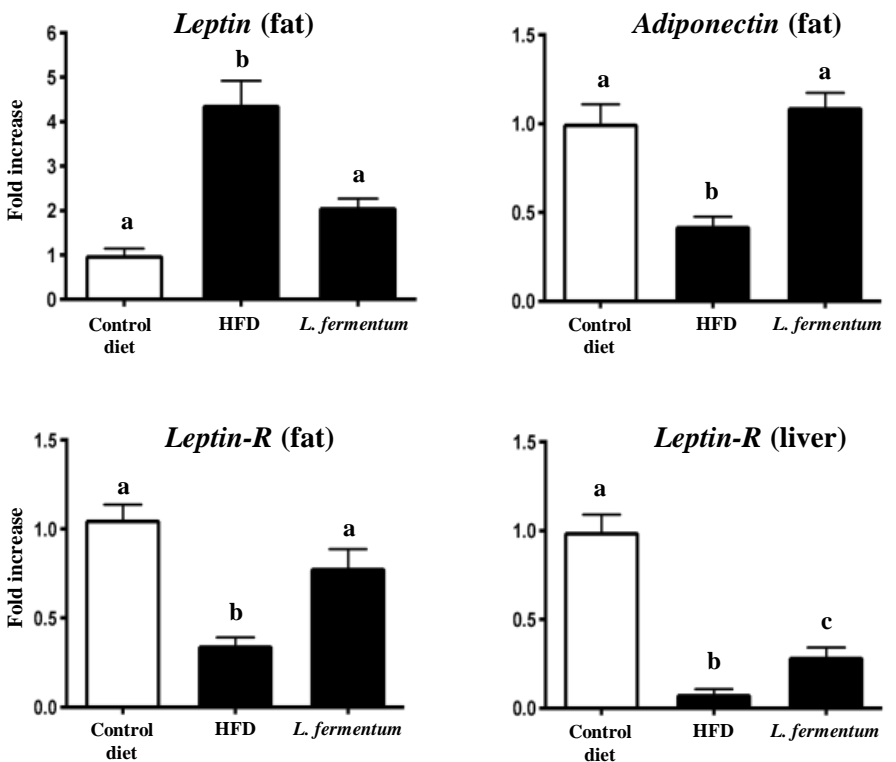
Figure 5

a)
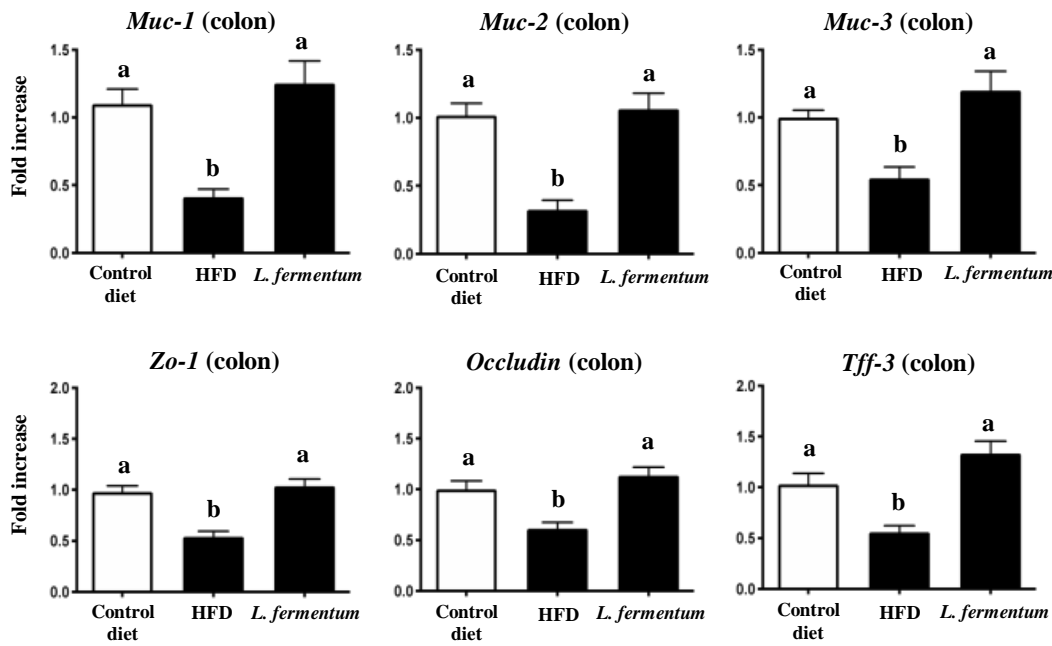

b)

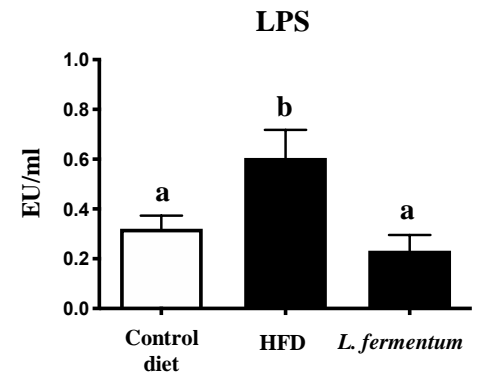


Figure 6

a)
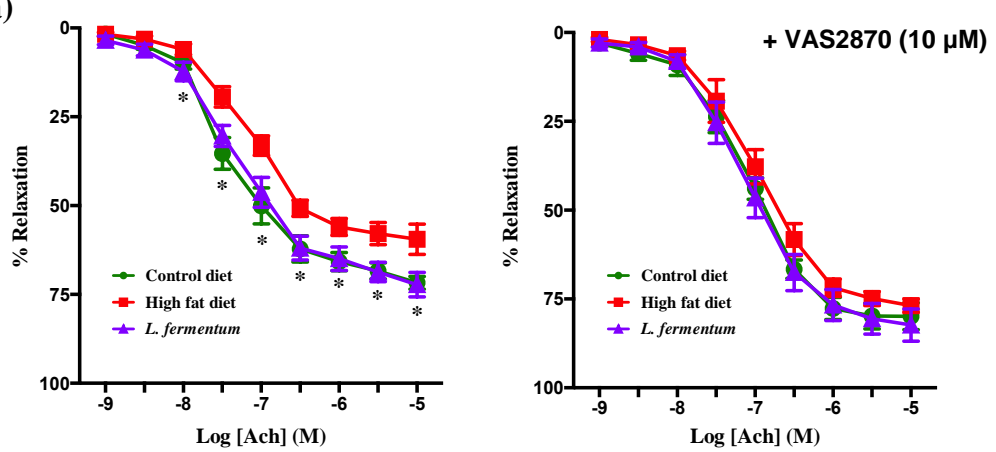

b)
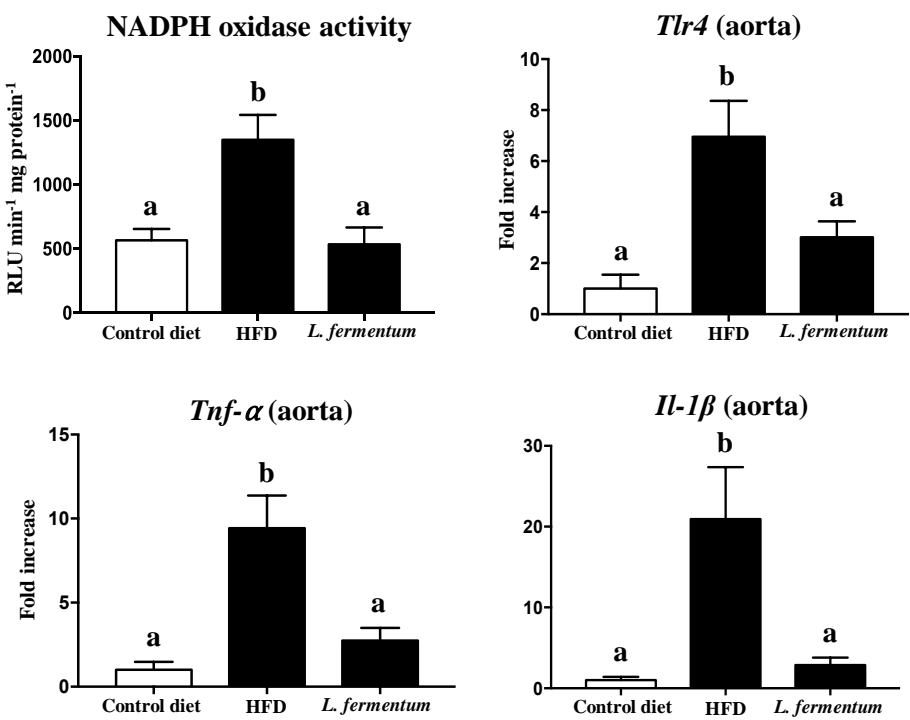
Figure 7

a)

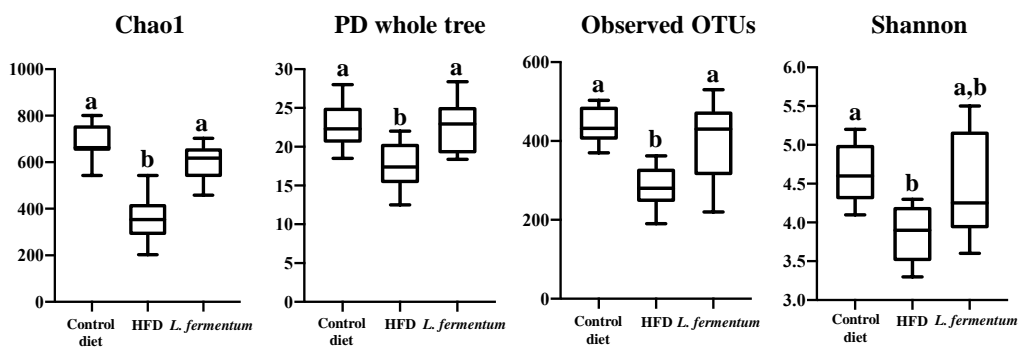

b)
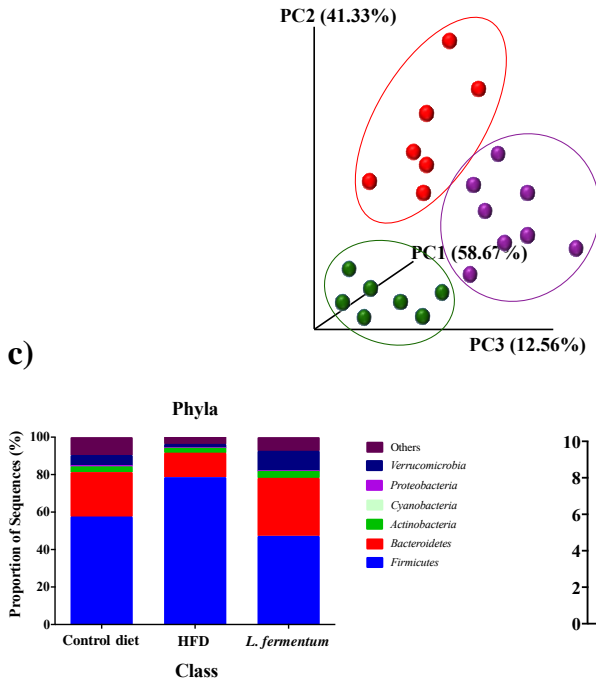

- Control diet

- High fat diet

L. fermentum

c)
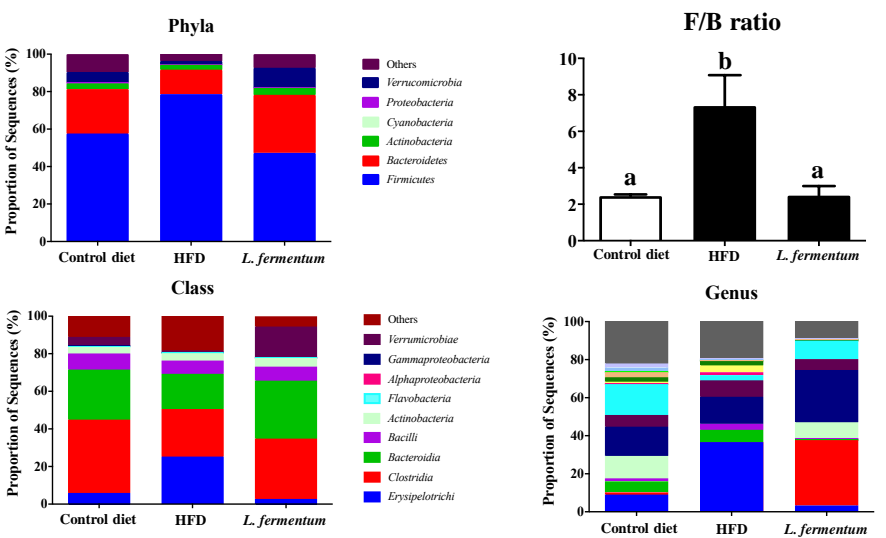

E- Oher

Derived form Lachnospiraceae

Enterorhabdis

Coploteremes

- Eubacterium

Lactobacilltus

- Bacteroides

- Sisymbrium

Derived fom

- Akkemanan 
Figure S1

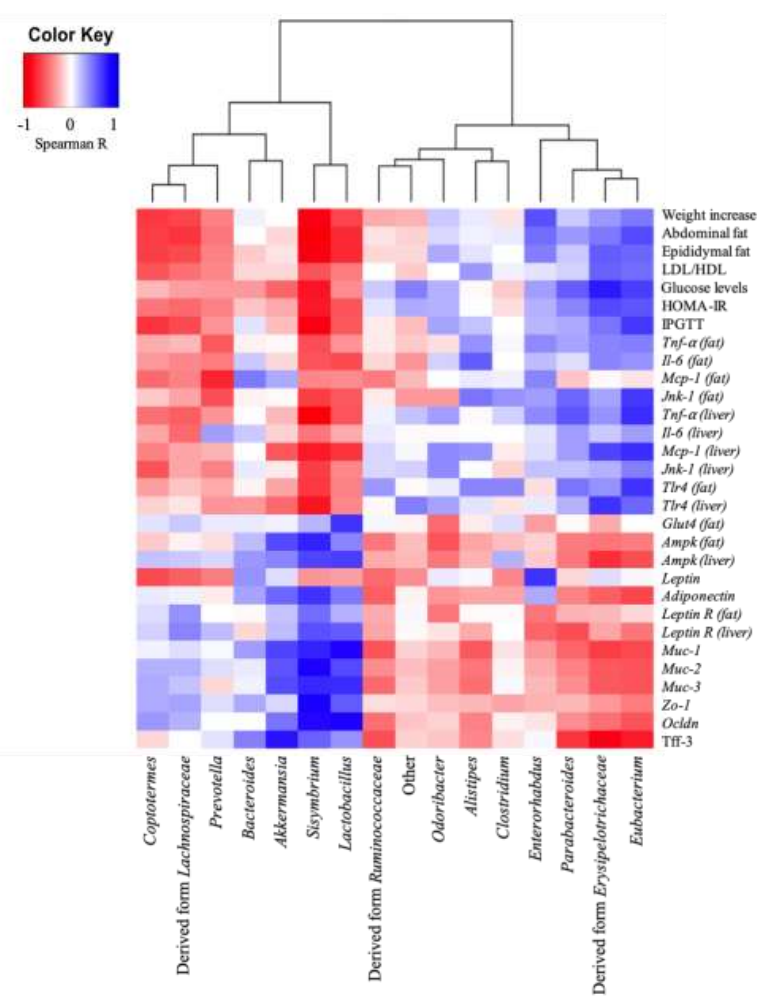


Figure 8

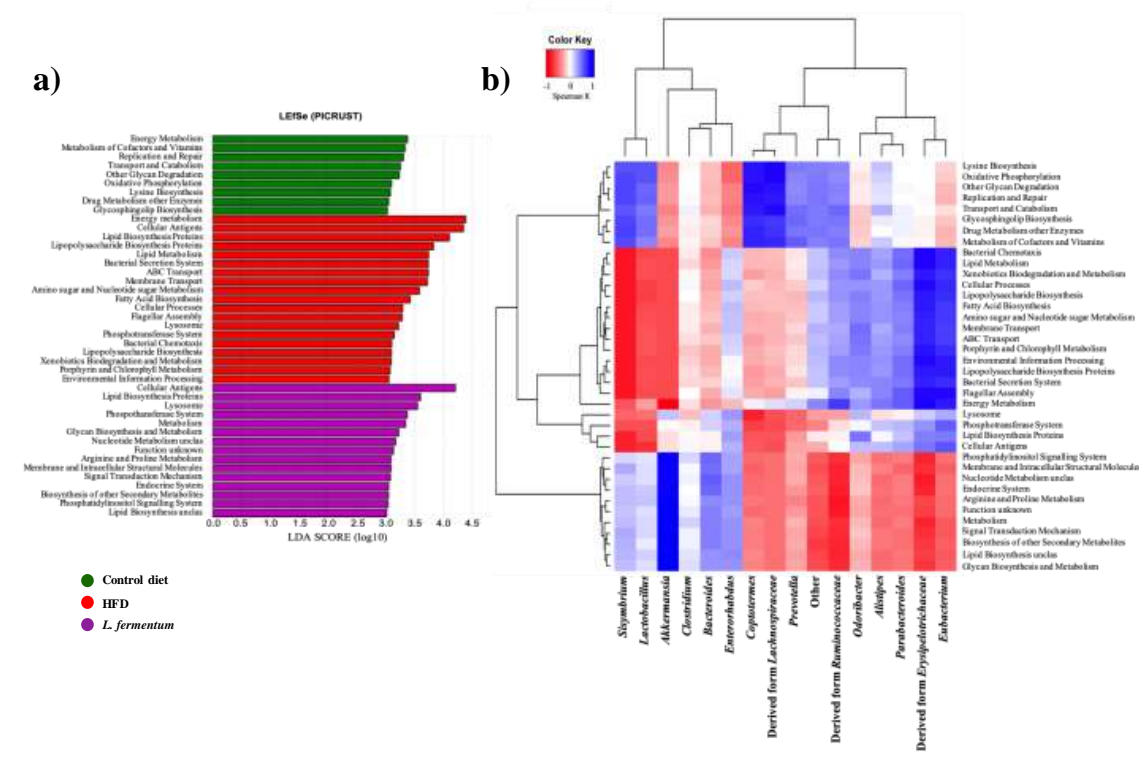

c)

a) Control diet

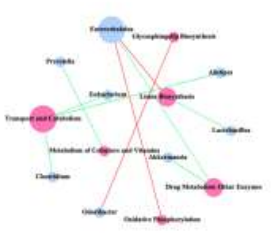

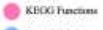

b) HFD

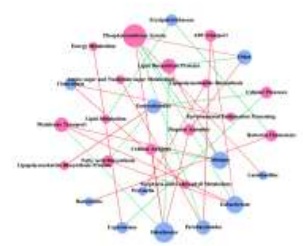

c) L. fermentum

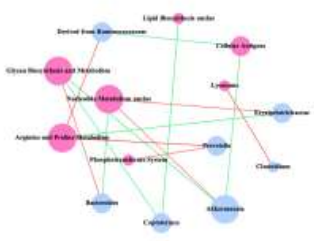

